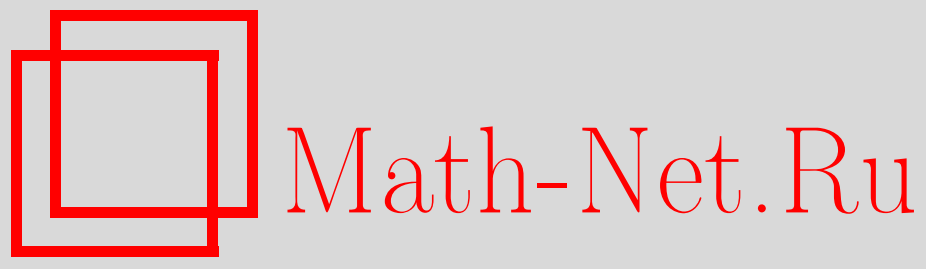

А. В. Покровский, Устранимые особенности решений уравнения минимальных поверхностей, Функи. анализ и его прил., 2005, том 39, выпуск 4, 62-68

DOI: https://doi.org/10.4213/faa85

Использование Общероссийского математического портала Math-Net.Ru подразумевает, что вы прочитали и согласны с пользовательским соглашением

http://www. mathnet.ru/rus/agreement

Параметры загрузки:

IP : 35.174 .16 .151

26 апреля 2023 г., 16:50:44

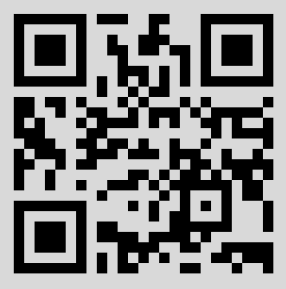


Функииональный анализ и его приложения

2005, т. 39, вып. 4, с. $62-68$

УДК 517.956

\title{
Устранимые особенности решений уравнения минимальных поверхностей*
}

\author{
(c) 2005. А. В. Покровский
}

\section{Введение}

В настоящей работе рассматривается задача о «стирании» особенностей для решений уравнения минимальных поверхностей

$$
\operatorname{div}\left(\left(1+|\nabla f|^{2}\right)^{-1 / 2} \nabla f\right)=0,
$$

$\nabla f=\left(D_{1} f, \ldots, D_{n} f\right)=\left(\partial f / \partial x_{1}, \ldots, \partial f / \partial x_{n}\right)$. Решением такого уравнения на непустом открытом множестве $G \subset \mathbb{R}^{n}(n \geqslant 2)$ называется, как обычно, функция $f \in C^{2}(G)$, для которой всюду в $G$ выполнено равенство (1). Множество всех таких $f$ мы обозначаем через $M(G)$.

Пусть $G$ - область в $\mathbb{R}^{n}$, которая в дальнейшем всегда предполагается ограниченной, $E$ - замкнутое множество в $G, E \neq G$, и $\alpha \in(0,1)$, и пусть $C_{\text {loc }}^{1, \alpha}(G)-$ множество всех непрерывно дифференцируемых функций в $G$, все частные производные которых удовлетворяют условию Гёльдера с показателем $\alpha$ на каждом компактном подмножестве области $G$. Множество $E$ называется устранимым для решений уравнения минимальных поверхностей в классе $C_{\mathrm{loc}}^{1, \alpha}(G)$, если для каждой функции $f \in C_{\mathrm{loc}}^{1, \alpha}(G)$, дважды непрерывно дифференцируемой на $G \backslash E$ и удовлетворяющей там равенству (1), существует такая функция $g \in M(G)$, что $f \equiv g$ на $G \backslash E$.

В принятых выше обозначениях сформулируем основной результат работы.

ТЕОрема 1. Для того чтобы множество Е было устранимым для решений уравнения минимальных поверхностей в классе $C_{\mathrm{loc}}^{1, \alpha}(G)$, необходимо и достаточно, чтобы оно имело нулевую хаусдорфову меру порядка $n-1+\alpha$, m. е. mes $^{n-1+\alpha} E=0$.

Остановимся на истории вопроса. Хорошо известно, что условие mes $^{n-1} E=0$ является достаточным для того, чтобы всякая функция $f \in M(G \backslash E)$ продолжалась на $E$ до функции из класса $M(G)$. Подчеркнем, что здесь не требуется никаких условий на поведение $f$ вблизи $E$. Для случая, когда множество $E$ состоит только из изолированных точек, этот результат был установлен Берсом [1], при $n=2$ - Ниче [2], для компактных множеств $E-$ Де Джорджи и Стампаккья [3], а в общем случае - Мирандой [4] (см. также [5, теорема 16.9]; другие доказательства даны в работах [6,7]). Заметим, что аналогичный результат имеет место для голоморфных функций нескольких комплексных переменных: если $G$ - область в $\mathbb{C}^{n}(n \geqslant 2)$ и $E$ - замкнутое множество в $G$

*Эта работа выполнена при частичной поддержке Государственной программы Украины №0102U000917. 
с $\operatorname{mes}^{2 n-2} E=0$, то любая функция $f$, голоморфная в $G \backslash E$, допускает продолжение на $E$ до функции, голоморфной в $G$ (см. [8]). С другой стороны, для гармонических функций теоремы подобного типа, очевидно, не справедливы: нужна дополнительная информация о поведении функции $f$ вблизи множества $E$. Приведем один из таких результатов, принадлежащий Е. П. Долженко (см. $[9,10]):$ для того чтобы любая функция $f \in C_{\mathrm{loc}}^{1, \alpha}(G)$, гармоническая на множестве $G \backslash E$, продолжалась с $G \backslash E$ до функции, гармонической в $G$, необходимо и достаточно, чтобы $E$ имело нулевую хаусдорфову меру порядка $n-1+\alpha$. Заметим, что для голоморфных функций более чем одного комплексного переменного результаты подобного типа не имеют места - ввиду теоремы Хартогса об устранимости компактных особенностей (см. [8]). Таким образом, теорема 1 является аналогом сформулированной теоремы Долженко об устранимых особенностях гармонических функций для решений уравнения минимальных поверхностей; в сочетании с другими приведенными выше результатами она показывает, что особые множества решений уравнения (1) наследуют свойства особых множеств как голоморфных функций нескольких комплексных переменных, так и гармонических функций.

Работа организована следующим образом. В первом параграфе напоминаются необходимые определения и приводится один вспомогательный результат, второй параграф посвящен доказательству теоремы 1.

\section{§1. Определения и вспомогательные результаты}

Всюду ниже выражения вида $C(\beta, \gamma, \ldots)$ обозначают неотрицательные величины, зависящие лишь от $\beta, \gamma, \ldots$; при этом в разных формулах величины с одним и тем же обозначением, вообе говоря, различаются между собой.

Если $x \in \mathbb{R}^{n}$ и $r>0$, то $|x|$ обозначает евклидову норму вектора $x, B(x, r):=$ $\left\{y \in \mathbb{R}^{n}:|y-x|<r\right\}$ и $B(0, r)=: B_{r}$.

Как обычно, $L_{2}(G)$ - пространство всех функций $f$, суммируемых с квадратом в области $G$ (здесь и далее рассматриваются измеримые в $G$ функции, принимающие только вещественные значения), с нормой $\left\|f \mid L_{2}(G)\right\|:=$ $\left(\int_{G}|f(x)|^{2} d x\right)^{1 / 2}, W^{1,2}(G)$ - соболевское пространство всех функций $f \in L_{2}(G)$, имеющих обобщенные производные $D_{i} f \in L_{2}(G), i=1, \ldots, n$, с нормой $\left\|f\left|W^{1,2}(G)\|:=\| f\right| L_{2}(G)\right\|+\sum_{i=1}^{n}\left\|D_{i} f \mid L_{2}(G)\right\|, W_{0}^{1,2}(G)$ - замыкание по этой норме пространства $C_{0}^{\infty}(G)$ всех бесконечно дифференцируемых функций с компактным носителем в $G, C^{1}(G)$ и $C^{1}(\bar{G})$ - соответственно пространство всех функций $f$, непрерывно дифференцируемых в $G$, и его подпространство, состоящее из тех $f$, для которых $f$ и $\nabla f$ непрерывно продолжаются на замкнутую область $\bar{G}$ (норма функции $f$ в этих пространствах определяется формулой $\left.\left\|f\left|C^{1}(\bar{G})\|:=\| f\right| C^{1}(G)\right\|:=\sup _{G}|f|+\sum_{i=1}^{n} \sup _{G}\left|D_{i} f\right|\right)$. Если $0<\alpha<1$, то $C^{\alpha}(G)$ - пространство всех $f \in C(G)$, для которых конечна норма

$$
\left\|f\left|C^{\alpha}(G) \|:=\sup _{G}\right| f \mid+\sup _{x, y \in G, x \neq y} \frac{|f(x)-f(y)|}{|x-y|^{\alpha}}\right.
$$

а $C^{1, \alpha}(G)$ - пространство всех $f \in C^{1}(G)$, для которых конечна норма

$$
\left\|f\left|C^{1, \alpha}(G)\left\|:=\sup _{G}|f|+\sum_{i=1}^{n}\right\| D_{i} f\right| C^{\alpha}(G)\right\| .
$$


Каждая функция $f \in C^{1, \alpha}(G)$ по непрерывности продолжается вместе со своим градиентом на $\bar{G}$; множество всех таких продолженных функций обозначается через $C^{1, \alpha}(\bar{G})$

Пусть $E \subset \mathbb{R}^{n}, \gamma>0$. Напомним, что (внешней) мерой Хаусдорфа mеs ${ }^{\gamma} E$ множества $E$ порядка $\gamma$ называется конечный или равный $+\infty$ предел при $t \rightarrow 0$ величины $\inf \left(\sum_{i} r_{i}^{\gamma}\right)$, где инфимум берется по всем не более чем счетным множествам открытых шаров $\left\{B\left(x_{i}, r_{i}\right)\right\}_{i}$ с $r_{i} \leqslant t$, образующим покрытие множества $E$.

В доказательстве теоремы 1 будет использовано следующее вспомогательное утверждение. Для его формулировки напомним, что двоичным кубом в $\mathbb{R}^{n}$ называется всякий куб вида $\left[s_{1} 2^{-k},\left(s_{1}+1\right) 2^{-k}\right] \times \cdots \times\left[s_{n} 2^{-k},\left(s_{n}+1\right) 2^{-k}\right]$, где $s_{1}, \ldots, s_{n}$ - целые числа, а $k$ - натуральное число. Если $x \in \mathbb{R}^{n}, r>0$, то $Q(x, r)$ обозначает замкнутый куб с центром в $x$, ребра которого параллельны координатным осям, а их длина равна $2 r$. При $t>0$ мы полагаем $t Q(x, r):=Q(x, t r)$.

ЛЕмма 1 (см. [11; 12, с. 20-22]). Пусть $\left\{Q_{l}\right\}_{l=1}^{m}(m \in \mathbb{N})-$ конечное множество двоичных кубов, внутренности которых попарно не пересекаются, $Q_{l}=Q\left(x_{l}, r_{l}\right), l=1, \ldots, m$. Тогда существует такое множество функиий $\left\{\varphi_{l}\right\}_{l=1}^{m} \subset C_{0}^{\infty}\left(\mathbb{R}^{n}\right)$, принимающих значения в интервале $[0,1]$, что носитель функции $\varphi_{l}$ содержится в кубе $\frac{3}{2} Q_{l}, \sup _{\frac{3}{2} Q_{l}}\left|\nabla \varphi_{l}\right| \leqslant C(n) r_{l}^{-1}(l=1, \ldots, m)$ u на множестве $\bigcup_{l=1}^{m} Q_{l}$ выполняется равенство $\sum_{l=1}^{m} \varphi_{l}(x)=1$.

\section{§2. Доказательство теоремы 1}

Пусть mes $^{n-1+\alpha} E=0$, а $f$ - функция из класса $C^{1, \alpha}(G)_{\text {lос }}$, дважды непрерывно дифференцируемая на множестве $G \backslash E$ и удовлетворяющая там равенству (1). Покажем, что $f \in M(G)$. Это равносильно тому (см., например, [5]), что $f$ является слабым решением уравнения $(1)$ в $G$, т.е. для произвольной функции $\varphi \in C_{0}^{\infty}(G)$ выполняется равенство

$$
M_{f}(\varphi):=\int_{G}\left(1+|\nabla f|^{2}\right)^{-1 / 2} \nabla f \cdot \nabla \varphi d x=0,
$$

где $\nabla f \cdot \nabla \varphi=\sum_{i=1}^{n} D_{i} f D_{i} \varphi$. Не ограничивая общности, можно, очевидно, считать, что $f \in C^{1, \alpha}(G)$.

Зафиксируем произвольным образом $\varepsilon>0$ и функцию $\varphi \in C_{0}^{\infty}(G)$. Поскольку $E \cap \operatorname{supp} \varphi-$ компакт, то из определения хаусдорфовой меры и того, что каждый шар содержится в объединении не более чем $5^{n}$ двоичных кубов, длина ребра которых не превосходит радиуса этого шара, вытекает существование такого конечного множества двоичных кубов $\left\{Q_{l}\right\}_{l=1}^{m}(m \in \mathbb{N}), Q_{l}=Q\left(x_{l}, r_{l}\right)$, $l=1, \ldots, m$, внутренности которых попарно не пересекаются, что $E \cap \operatorname{supp} \varphi \subset$ $\bigcup_{l=1}^{m} Q_{i} \subset \bigcup_{l=1}^{m} \frac{3}{2} Q_{l} \subset G$ и $\sum_{l=1}^{m} r_{l}^{n-1+\alpha} \leqslant \varepsilon$. Пусть $\left\{\varphi_{l}\right\}_{l=1}^{m}-$ множество функций из леммы 1 , образующих разбиение единицы для системы кубов $\left\{Q_{l}\right\}_{l=1}^{m}$. Из включения $f \in C^{1, \alpha}(G)$ следует, что $\left(1+|\nabla f|^{2}\right)^{-1 / 2} \nabla f \in C^{\alpha}(G)$, откуда, пользуясь оценкой производных функций $\varphi_{l}$ из леммы 1 и тем, что носитель функции $\varphi\left(1-\sum_{l=1}^{m} \varphi_{l}\right)$ содержится в $G \backslash E\left(\right.$ и, следовательно, $\left.M_{f}\left(\varphi\left(1-\sum_{l=1}^{m} \varphi_{l}\right)\right)=0\right)$, 
получаем

$$
\begin{aligned}
\left|M_{f}(\varphi)\right| & =\left|\sum_{l=1}^{m} M_{f}\left(\varphi \varphi_{l}\right)+M_{f}\left(\varphi\left(1-\sum_{l=1}^{m} \varphi_{l}\right)\right)\right| \leqslant \sum_{l=1}^{m}\left|M_{f}\left(\varphi \varphi_{l}\right)\right| \\
& =\sum_{l=1}^{m}\left|\int_{G}\left(1+|\nabla f|^{2}\right)^{-1 / 2} \nabla f \cdot \nabla\left(\varphi \varphi_{l}\right) d x\right| \\
& =\sum_{l=1}^{m}\left|\int_{\frac{3}{2} Q_{l}}\left(1+|\nabla f|^{2}\right)^{-1 / 2} \nabla f \cdot \nabla\left(\varphi \varphi_{l}\right) d x\right| \\
& =\sum_{l=1}^{m}\left|\int_{\frac{3}{2} Q_{l}}\left(\left(1+|\nabla f|^{2}\right)^{-1 / 2} \nabla f-\left(1+\left|\nabla f\left(x_{l}\right)\right|^{2}\right)^{-1 / 2} \nabla f\left(x_{l}\right)\right) \cdot \nabla\left(\varphi \varphi_{l}\right) d x\right| \\
& \leqslant \sum_{l=1}^{m} C(f, \varphi, G, n, \alpha) r_{l}^{\alpha} r_{l}^{-1} r_{l}^{n}=C(f, \varphi, G, n, \alpha) \sum_{l=1}^{m} r_{l}^{\alpha} r_{l}^{-1} r_{l}^{n} \\
& \leqslant C(f, \varphi, G, n, \alpha) \varepsilon .
\end{aligned}
$$

Поскольку $\varepsilon$ и $\varphi$ выбирались произвольным образом, это означает, что $M_{f}(\varphi)=0$ и, следовательно, $f \in M(G)$.

Достаточность условия теоремы 1 установлена.

Докажем необходимость.

Пусть mes $^{n-1+\alpha} E>0$. Поскольку область $G$ ограничена, то, не умаляя общности, можно считать, что $G=B_{R}$ при некотором $R>0$, а выделяя в случае необходимости подмножество, - что $E \Subset B_{R}$. По лемме Фростмана (см. [13]) существует такая борелевская мера $\mu$ с носителем на $E$, что $\mu(E)>0$ и $\mu(B(x, r)) \leqslant r^{n-1+\alpha}$ для каждого шара $B(x, r) \subset \mathbb{R}^{n}$.

Обозначим через $B_{R}^{1, \alpha}$ единичный шар в пространстве $C^{1, \alpha}\left(\bar{B}_{R}\right)$.

Пусть $t>0$ и $f \in B_{R}^{1, \alpha}$. Рассмотрим функцию $g_{t} \in W_{0}^{1,2}\left(B_{R}\right)$, которая является решением неоднородного уравнения

$$
\operatorname{div}\left(\left(1+|\nabla f|^{2}\right)^{-1 / 2} \nabla g_{t}\right)=t \mu .
$$

Равенство (2) понимается в обобщенном смысле, оно означает, что для произвольной функции $\varphi \in C_{0}^{\infty}\left(B_{R}\right)$ выполняется тождество

$$
-\int_{B_{R}}\left(1+|\nabla f|^{2}\right)^{-1 / 2} \nabla g_{t} \cdot \nabla \varphi d x=t \int \varphi d \mu .
$$

Функция $g_{t}$ всегда существует, единственна и представляется в виде $g_{t}(x)=$ $t \int_{B_{R}} G_{f}(x, y) d \mu$, где $G_{f}(x, y)$ - функция Грина для равномерно эллиптического оператора $L_{f} u=\operatorname{div}\left(\left(1+|\nabla f|^{2}\right)^{-1 / 2} \nabla u\right)$ в шаре $B_{R}$ (см. [14]). Покажем, что при малых $t$ отображение $A_{t}$, переводящее $f$ в функцию $g_{t}=: A_{t} f$, будет отображением множества $B_{R}^{1, \alpha}$ в себя. Поскольку $A_{t}=t A$, где $A:=A_{1}$, то достаточно установить, что $g:=A f \in C^{1, \alpha}\left(B_{R}\right)$ и

$$
\sup \left\{\left\|A f \mid C^{1, \alpha}\left(B_{R}\right)\right\|: f \in B_{R}^{1, \alpha}\right\}<\infty .
$$

Для этого воспользуемся приемом, предложенным в [15]. Пусть $h$ - образ функции, тождественно равной нулю, при отображении $A$ и $u=\nabla h$. Тогда $h$ гармо-

Функциональный анализ и его приложения, т. 39, вып. 4 
нична в $B_{R} \backslash E$, принадлежит классу $C^{1, \alpha}\left(\bar{B}_{R}\right)$ (см. [10,15]), и, следовательно, $u \in C^{\alpha}\left(B_{R}\right)$, а уравнение (2) при $t=1$ равносильно уравнению

$$
\operatorname{div}\left(\left(1+|\nabla f|^{2}\right)^{-1 / 2} \nabla g\right)=\operatorname{div} u .
$$

Элементарно проверяется, что $\left(1+|\nabla f|^{2}\right)^{-1 / 2} \in C^{\alpha}\left(B_{R}\right)$ и

$$
\left\|\left(1+|\nabla f|^{2}\right)^{-1 / 2}\left|C^{\alpha}\left(B_{R}\right)\|\leqslant 1+\| f\right| C^{1, \alpha}\left(B_{R}\right)\right\| \leqslant 2 .
$$

Отсюда следует (см. [16, теоремы 8.33, 8.34], что $g \in C^{1, \alpha}\left(\bar{B}_{R}\right)$ и

$$
\left\|g \mid C^{1, \alpha}\left(B_{R}\right)\right\| \leqslant C(n, R)\left(\sup _{B_{R}}|g|+\left\|u \mid C^{\alpha}\left(B_{R}\right)\right\|\right) .
$$

С другой стороны (см. [16, теорема 8.16]), имеет место оценка

$$
\sup _{B_{R}}|g| \leqslant C(n, R) \sup _{B_{R}}|u| .
$$

Объединяя (5) и (6), получаем

$$
\left\|g\left|C^{1, \alpha}\left(B_{R}\right)\|\leqslant C(n, R)\| u\right| C^{\alpha}\left(B_{R}\right)\right\| \leqslant C(n, R)\left\|h \mid C^{1, \alpha}\left(B_{R}\right)\right\|,
$$

откуда вытекает (4). Следовательно, существует такое $t>0$, что $A_{t}$ отображает шар $B_{R}^{1, \alpha}$ в себя. Зафиксируем такое $t$ и покажем, что отображение $A_{t}$ непрерывно по норме пространства $C^{1}\left(\bar{B}_{R}\right)$.

Пусть последовательность $\left\{f_{i}\right\}_{i=1}^{\infty}$ функций из $B_{R}^{1, \alpha}$ сходится при $i \rightarrow \infty$ к функции $f$ по норме пространства $C^{1}\left(\bar{B}_{R}\right)$. Очевидно, что $f \in B_{R}^{1, \alpha}$. Докажем, что $A_{t} f_{i} \rightarrow A_{t} f$ при $i \rightarrow \infty$. Поскольку $A_{t}=t A$, то для этого достаточно установить, что $\left\|A f_{i}-A f \mid C^{1}\left(\bar{B}_{R}\right)\right\| \rightarrow 0$ при $i \rightarrow \infty$. Из определения функций $A f_{i}$ и $A f$ следует, что для каждой функции $\varphi \in W_{0}^{1,2}\left(B_{R}\right)$ справедливы равенства

$$
\begin{aligned}
0= & \int_{B_{R}}\left(1+\left|\nabla f_{i}\right|^{2}\right)^{-1 / 2} \nabla\left(A f_{i}\right) \cdot \nabla \varphi d x-\int_{B_{R}}\left(1+|\nabla f|^{2}\right)^{-1 / 2} \nabla(A f) \cdot \nabla \varphi d x \\
= & \int_{B_{R}}\left(1+\left|\nabla f_{i}\right|^{2}\right)^{-1 / 2} \nabla\left(A f_{i}-A f\right) \cdot \nabla \varphi d x \\
& \quad-\int_{B_{R}}\left[\left(1+|\nabla f|^{2}\right)^{-1 / 2}-\left(1+\left|\nabla f_{i}\right|^{2}\right)^{-1 / 2}\right] \nabla(A f) \cdot \nabla \varphi d x,
\end{aligned}
$$

откуда вытекает, что

$$
\begin{aligned}
\int_{B_{R}} & \left(1+\left|\nabla f_{i}\right|^{2}\right)^{-1 / 2} \nabla\left(A f_{i}-A f\right) \cdot \nabla \varphi d x \\
& =\int_{B_{R}}\left[\left(1+|\nabla f|^{2}\right)^{-1 / 2}-\left(1+\left|\nabla f_{i}\right|^{2}\right)^{-1 / 2}\right] \nabla(A f) \cdot \nabla \varphi d x .
\end{aligned}
$$

Подставляя сюда $\varphi=A f_{i}-A f$, приходим к равенству

$$
\begin{aligned}
\int_{B_{R}} & \left(1+\left|\nabla f_{i}\right|^{2}\right)^{-1 / 2}\left|\nabla\left(A f_{i}-A f\right)\right|^{2} d x \\
& =\int_{B_{R}}\left[\left(1+|\nabla f|^{2}\right)^{-1 / 2}-\left(1+\left|\nabla f_{i}\right|^{2}\right)^{-1 / 2}\right] \nabla(A f) \cdot \nabla\left(A f_{i}-A f\right) d x .
\end{aligned}
$$


Пользуясь неравенством Гёльдера и тем, что $\sup _{B_{R}}|\nabla f| \leqslant 1$, получаем из (7) следующую цепочку неравенств:

$$
\begin{aligned}
\frac{1}{\sqrt{2}} \int_{B_{R}}\left|\nabla\left(A f_{i}-A f\right)\right|^{2} d x \leqslant \int_{B_{R}}\left(1+\left|\nabla f_{i}\right|^{2}\right)^{-1 / 2}\left|\nabla\left(A f_{i}-A f\right)\right|^{2} d x \\
\leqslant\left(\sup _{B_{R}}\left|\left(1+|\nabla f|^{2}\right)^{-1 / 2}-\left(1+\left|\nabla f_{i}\right|^{2}\right)^{-1 / 2}\right|\right)\left\|\nabla(A f) \mid L_{2}\left(B_{R}\right)\right\| \\
\quad \times\left\|\nabla\left(A f_{i}-A f\right) \mid L_{2}\left(B_{R}\right)\right\|,
\end{aligned}
$$

откуда следует, что

$$
\begin{aligned}
& \left\|\nabla\left(A f_{i}-A f\right) \mid L_{2}\left(B_{R}\right)\right\| \\
& \quad \leqslant \sqrt{2}\left(\sup _{B_{R}}\left|\left(1+|\nabla f|^{2}\right)^{-1 / 2}-\left(1+\left|\nabla f_{i}\right|^{2}\right)^{-1 / 2}\right|\right)\left\|\nabla(A f) \mid L_{2}\left(B_{R}\right)\right\| .
\end{aligned}
$$

Если $i \rightarrow \infty$, то $f_{i} \rightarrow f$ в $C^{1}\left(\bar{B}_{R}\right)$ и правая часть формулы (8) стремится к нулю. Применяя неравенство Пуанкаре $\left\|\varphi\left|L_{2}\left(B_{R}\right)\|\leqslant C(n) R\| \nabla \varphi\right| L_{2}\left(B_{R}\right)\right\|$ (для всех $\left.\varphi \in W_{0}^{1,2}\left(B_{R}\right)\right)$, отсюда заключаем, что $A f_{i} \rightarrow A f$ в $W^{1,2}\left(B_{R}\right)$ при $i \rightarrow \infty$. $\mathrm{C}$ другой стороны, по теореме Арцела-Асколи последовательность $\left\{A f_{i}\right\}_{i=1}^{\infty}$ предкомпактна в $C^{1}\left(\bar{B}_{R}\right)$, откуда следует, что $A f_{i} \rightarrow A f$ в $C^{1}\left(\bar{B}_{R}\right)$. Это доказывает непрерывность отображения $A_{t}$.

Таким образом, $A_{t}$ является непрерывным отображением в себя выпуклого компактного множества $B_{R}^{1, \alpha}$ в банаховом пространстве $C^{1}\left(\bar{B}_{R}\right)$. По теореме Шаудера (см., например, [16, теорема 11.1]) это отображение имеет неподвижную точку $v \in B_{R}^{1, \alpha}$. Это означает, что $v$ удовлетворяет в обобщенном смысле равенству

$$
\operatorname{div}\left(\left(1+|\nabla v|^{2}\right)^{-1 / 2} \nabla v\right)=t \mu
$$

в шаре $B_{R}$. Следовательно, $v \in C^{1, \alpha}\left(B_{R}\right)$, сужение функции $v$ на $G \backslash E-$ функция из $M\left(B_{R} \backslash E\right)$, но $v \notin M\left(B_{R}\right)$.

Теорема 1 доказана.

\section{ЛитерАТУра}

1. Bers L. Isolated singularities of minimal surfaces. Ann. Math., 53, 364-386 (1951).

2. Nitsche J. C. C. On new results in the theory of minimal surfaces. Bull. Amer. Math. Soc., 71, 195-270 (1965).

3. De Giorgi E., Stampacchia G. Sulla singolarità eliminabili delle ipersuperficie minimali. Atti Accad. Naz. Lincei, Rend., Cl. Sci. Fis. Mat. Nat., 38, 352-357 (1965).

4. Miranda $M$. Sulla singolarità eliminabili delle soluzioni dell'equazione delle superfici minime. Ann. Sc. Norm. Sup. Pisa, Ser. IV, 4, 129-132 (1977).

5. Джусти Э. Минимальные поверхности и функции ограниченной вариации. Мир, M., 1989.

6. Anzellotti G. Dirichlet problem and removable singularities for functional with linear growth. Boll. Un. Mat. Ital. C (5), 81, 141-159 (1981).

7. Simon L. On a theorem of De Giorgi and Stampacchia. Math. Z., 155, 199-204 (1977).

8. Чирка E. M. Комплексные аналитические множества. Наука, М., 1985.

9. Долженко E. П. О представлении непрерывных гармонических функций в виде потенциалов. Изв. АН СССР, сер. матем., 28, №5, 1113-1130 (1964).

10. Долженко E. П. Об особых точках непрерывных гармонических функций. Изв. АН СССР, сер. матем., 28, №6, 1251-1270 (1964). 
11. Harvey R., Polking J. C. Removable singularities of solutions of linear partial differential equations. Acta Math., 125, №1/2, 39-56 (1970).

12. Тарханов Н. Н. Ряд Лорана для решений эллиптических систем. Новосибирск, Наука, 1991.

13. Карлесон Л. Избранные проблемы теории исключительных множеств. Мир, М., 1971.

14. Littman W., Stampaccia G., Weinberger H. F. Regular points for elliptic equations with discontinuous coefficients. Ann. Scuola. Norm. Sup. Pisa, 17, №3, 43-77 (1963).

15. Lieberman G. M. Sharp form of estimates for subsolutions and supersolutions of quasilinear elliptic equations involving measures. Commun. Partial Diff. Equations, 18, №7\&8, 1191-1212 (1993).

16. Гилбарг Д., Трудингер Н. Эллиптические дифференциальные уравнения с частными производными второго порядка. Наука, М., 1989.

Институт математики НАН Украины

e-mail: pokrovsk@imath.kiev.ua

Поступило в редакцию

6 мая 2004 г. 